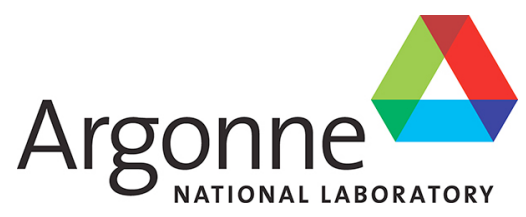

\title{
UNIC Code: Algorithmic Specification of the Method of Long Characteristics
}

Mathematics and Computer Science Division 


\begin{abstract}
About Argonne National Laboratory
Argonne is a U.S. Department of Energy laboratory managed by UChicago Argonne, LLC under contract DE-AC02-06CH11357. The Laboratory's main facility is outside Chicago, at 9700 South Cass Avenue, Argonne, Illinois 60439. For information about Argonne and its pioneering science and technology programs, see www.anl.gov.
\end{abstract}

\title{
DOCUMENT AVAILABILITY
}

Online Access: U.S. Department of Energy (DOE) reports produced after 1991 and a growing number of pre-1991 documents are available free via DOE's SciTech Connect (http://www.osti.gov/scitech/)

Reports not in digital format may be purchased by the public from the National Technical Information Service (NTIS):

U.S. Department of Commerce

National Technical Information Service

5301 Shawnee Rd

Alexandria, VA 22312

www.ntis.gov

Phone: (800) 553-NTIS (6847) or (703) 605-6000

Fax: (703) 605-6900

Email: orders@ntis.gov

Reports not in digital format are available to DOE and DOE contractors from the Office of Scientific and Technical Information (OSTI):

U.S. Department of Energy

Office of Scientific and Technical Information

P.O. Box 62

Oak Ridge, TN 37831-0062

www.osti.gov

Phone: (865) 576-8401

Fax: (865) 576-5728

Email: reports@osti.gov

\footnotetext{
Disclaimer

This report was prepared as an account of work sponsored by an agency of the United States Government. Neither the United States Government nor any agency thereof, nor UChicago Argonne, LLC, nor any of their employees or officers, makes any warranty, express or implied, or assumes any legal liability or responsibility for the accuracy, completeness, or usefulness of any information, apparatus, product, or process disclosed, or represents that its use would not infringe privately owned rights. Reference herein to any specific commercial product, process, or service by trade name, trademark, manufacturer, or otherwise, does not necessarily constitute or imply its endorsement, recommendation, or favoring by the United States Government or any agency thereof. The views and opinions of document authors expressed herein do not necessarily state or reflect those of the United States Government or any agency thereof, Argonne National Laboratory, or UChicago Argonne, LLC.
} 
ANL/MCS-TM-301

\section{UNIC Code: Algorithmic Specification of the Method of Long Characteristics}

prepared by

S. F. Siegel, A. R. Siegel, C. Rabiti

Mathematics and Computer Science Division, Argonne National Laboratory

June 2008 


\title{
UNIC Code: Algorithmic Specification of the Method of Long Characteristics
}

Technical Memorandum ANL/MCS-TM-301, June 2008

Division of Mathematics and Computer Science

Argonne National Laboratory

\section{Stephen F. Siegel}

Department of Computer and Information Sciences

103 Smith Hall

University of Delaware

Newark, DE 19716, USA

siegel@cis.udel.edu

\author{
Andrew R. Siegel \\ Argonne National Laboratory \\ 9700 S. Cass Avenue \\ Argonne, IL 60439, USA \\ siegela@mcs.anl.gov

\section{Cristian Rabiti} \\ Nuclear Engineering Division \\ Argonne National Laboratory \\ 9700 S. Cass Avenue \\ Argonne, IL 60439, USA \\ crabiti@anl.gov
}

Mathematics and Computer Science Division 
This page intentionally left blank. 


\section{Contents}

Abstract 1

1. The Steady State Neutron Transport Equation 2

1.1. Fundamental Equations 2

1.2. Energy Discretization 2

2. Geometry 3

2.1. Spatial Discretization 3

2.2. Integral Approximations 4

2.3. Boundary Condition 5

3. Outer Algorithm 5

4. Middle Algorithms 6

5. Inner Algorithm 8

6. Ray-tracing Algorithm 9

7. Conformance 12

References 12 
Abstract

The purpose of this document is to specify the method of long characteristics algorithm used in the UNIC neutron transport code. 


\section{The Steady State Neutron Transport Equation}

1.1. Fundamental Equations. In this section we briefly review the fundamental integrodifferential equation that we wish to solve. It is beyond the scope of this paper to describe the equation and its physical significance in detail. Such details are covered e.g. in [1].

Given $\sigma, \sigma^{s}, \sigma^{f}, \chi$, and $\nu$, we wish to solve the steady state neutron transport equation for $K_{\text {eff }}$ and $\psi$ :

$$
\begin{aligned}
{[\hat{\Omega} \cdot \vec{\nabla}+\sigma(\vec{r}, E)] \psi(\vec{r}, \hat{\Omega}, E)=} & \int d E^{\prime} \int d \hat{\Omega}^{\prime} \sigma^{s}\left(\vec{r}, E^{\prime} \rightarrow E, \hat{\Omega}^{\prime} \cdot \hat{\Omega}\right) \psi\left(\vec{r}, \hat{\Omega}^{\prime}, E^{\prime}\right) \\
& +\frac{\chi(E)}{4 \pi K_{e f f}} \int d E^{\prime} \nu \sigma^{f}\left(\vec{r}, E^{\prime}\right) \int d \hat{\Omega}^{\prime} \psi\left(\vec{r}, \hat{\Omega}^{\prime}, E^{\prime}\right)
\end{aligned}
$$

The notation is summarized in Figure 1. Notice that both sides of (1) are functions of $\vec{r}$, $\hat{\Omega}$, and $E$. The integral over energy $\left(E^{\prime}\right)$ runs from 0 to $\infty$; the integral over angle $\hat{\Omega}^{\prime}$ is an integral over the surface of the unit sphere $S^{2}$ (which has area $4 \pi$ ).

For simplicity in this version of the specification we assume isotropic scattering: i.e., $\sigma^{s}$ is independent of $\mu$. Note that when modeling nuclear reactor cores (the principle application domain of UNIC) this is not necessarily a good assumption and instead we will require an expansion of the flux to at least several $P_{n}$ modes. This and other features will be included in a subsequent version of the specification.

For the isotropic scattering case we can write $\sigma^{s}\left(\vec{r}, E^{\prime} \rightarrow E\right)$ for $\sigma^{s}\left(\vec{r}, E^{\prime} \rightarrow E, \mu\right)$ for any $\mu$. Then (1) becomes

$$
\begin{aligned}
{[\hat{\Omega} \cdot \vec{\nabla}+\sigma(\vec{r}, E)] \psi(\vec{r}, \hat{\Omega}, E)=} & \int d E^{\prime} \sigma^{s}\left(\vec{r}, E^{\prime} \rightarrow E\right) \phi\left(\vec{r}, E^{\prime}\right) \\
& +\frac{\chi(E)}{K_{\text {eff }}} \int d E^{\prime} \nu \sigma^{f}\left(\vec{r}, E^{\prime}\right) \phi\left(\vec{r}, E^{\prime}\right)
\end{aligned}
$$

where

$$
\phi(\vec{r}, E)=\frac{1}{4 \pi} \int_{S^{2}} \psi(\vec{r}, \hat{\Omega}, E) d \hat{\Omega}
$$

is the normalized isotropic scalar flux. Notice that in this case the right hand side of (2) is independent of $\hat{\Omega}$.

1.2. Energy Discretization. We now assume the energy dimension has been discretized by specifying $G+1$ real numbers $E_{G}<E_{G-1}<\ldots<E_{1}<E_{0}$. For $1 \leq g \leq G$, energy group $g$ is defined to be the interval of real numbers $\left[E_{g}, E_{g-1}\right]$. We let

$$
\begin{aligned}
\psi_{g}(\vec{r}, \hat{\Omega}) & =\int_{g} d E \psi(\vec{r}, \hat{\Omega}, E) \\
\phi_{g}(\vec{r}) & =\frac{1}{4 \pi} \int d \hat{\Omega} \psi_{g}(\vec{r}, \hat{\Omega}) .
\end{aligned}
$$

Recasting (2) in terms of these discrete energy groups requires the calculation of multigroup cross sections. The simplest way to do this is to assume that for each $g$, there is a function $f(E)$ defined on $\left[E_{g}, E_{g-1}\right]$ such that

$$
\psi(\vec{r}, E, \hat{\Omega}) \approx f(E) \psi_{g}(\vec{r}, \hat{\Omega}) .
$$




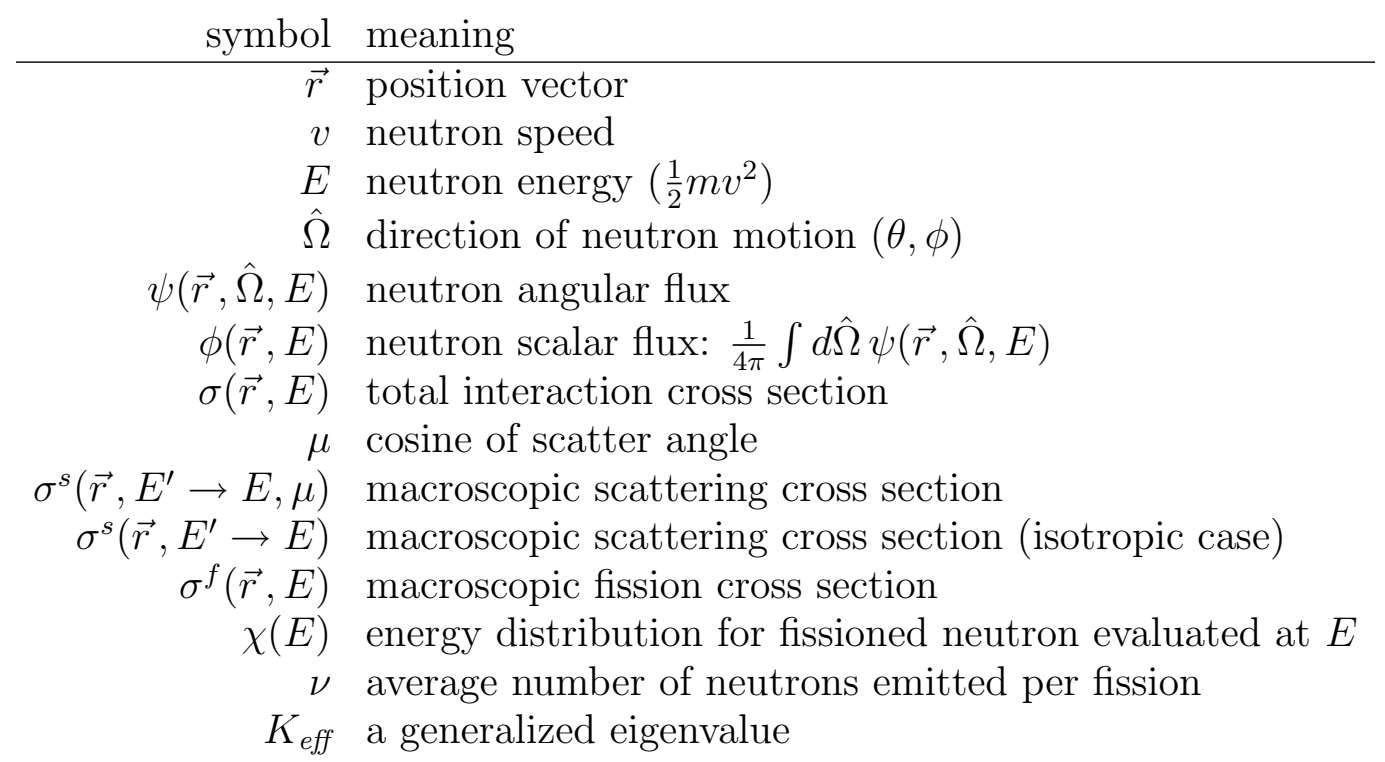

Figure 1. Symbols used in describing the linear Boltzman equation

Then define

$$
\begin{aligned}
\sigma_{g}(\vec{r}) & =\int_{g} d E \sigma(\vec{r}, E) f(E) \\
\sigma_{g}^{f}(\vec{r}) & =\int_{g} d E \sigma^{f}(\vec{r}, E) f(E) \\
\sigma_{g g^{\prime}}^{s}(\vec{r}, \mu) & =\int_{g} d E \int_{g^{\prime}} d E^{\prime} \sigma^{s}\left(\vec{r}, E^{\prime} \rightarrow E, \mu\right) f\left(E^{\prime}\right)
\end{aligned}
$$

To simplify the presentation, we shall just assume that suitably calculated multigroup cross sections are provided. For details, see [1, 2-2].

Discretizing (2), we arrive at one equation for each group $g$ :

$$
\left[\hat{\Omega} \cdot \vec{\nabla}+\sigma_{g}(\vec{r})\right] \psi_{g}(\vec{r}, \hat{\Omega})=\sum_{g^{\prime}} \sigma_{g g^{\prime}}^{s}(\vec{r}) \phi_{g^{\prime}}(\vec{r})+\frac{\chi_{g}}{K_{e f f}} \sum_{g^{\prime}} \nu \sigma_{g^{\prime}}^{f}(\vec{r}) \phi_{g^{\prime}}(\vec{r}) .
$$

\section{Geometry}

2.1. Spatial Discretization. We assume the domain is partitioned into $N$ distinct regions $V_{n}(1 \leq n \leq N)$, called elements. The volume of $V_{n}$ is vol $[n]$. The average scalar flux for group $g$ in $V_{n}$ is defined to be

$$
\phi_{g}[n]=\frac{1}{\operatorname{vol}[n]} \int_{V_{n}} \phi_{g}(\vec{r}) d V .
$$

The angular space, which can be identified with the unit sphere $S^{2}$, is partitioned into $I$ distinct "angular regions." For each $i, \hat{\Omega}_{i}$ will denote some fixed angle in angular region $i$. We let $\Delta \hat{\Omega}_{i}$ denote the quotient of the area (on the unit sphere) of the $i^{\text {th }}$ region by $4 \pi$. 
Since $4 \pi$ is the area of $S^{2}$, we have

$$
\sum_{i=1}^{I} \Delta \hat{\Omega}_{i}=1 .
$$

Moreover, the integral of a function $f$ defined on $S^{2}$ can be approximated as follows:

$$
\frac{1}{4 \pi} \int_{S^{2}} f(\hat{\Omega}) d \hat{\Omega} \approx \sum_{i=1}^{I} f_{i} \Delta \hat{\Omega}_{i}
$$

where $f_{i}$ is the representative value for $f$ in angular region $i$.

For each $i$, we are given $J_{i}$ trajectories (i.e., rays, also referred to as "tracks") in the direction $\hat{\Omega}_{i}$. The trajectories are numbered $1, \ldots, J_{i}$. Each trajectory has associated to it a trajectory cylinder with cross-sectional area $\Delta S_{i, j}$. One way to think of this is to associate to each $i$ a plane $P$ such that lines perpendicular to $P$ are oriented in the direction of $\hat{\Omega}_{i}$. Then a finite region of $P$ can be partitioned into $J_{i}$ disjoint subregions, with one starting point per subregion, and $\Delta S_{i, j}$ is the area of the $j^{\text {th }}$ such subregion. In any case, the volume of a "slice" of cylidner $j$ for angular region $i$ is the product of $\Delta S_{i, j}$ and the length of that slice.

Each trajectory enters the domain, passes through a sequence of elements, and then exits the domain. (We will assume the domain being modeled is convex so that it is not possible to exit and re-enter the domain.) We let $K_{i, j}$ denote the number of elements encountered by trajectory $j$ of angular region $i$, and we let $\iota(i, j, k)\left(1 \leq k \leq K_{i, j}\right)$ denote the index $n$ of the $k^{t h}$ element intersected. The length of the intersection of the trajectory with $V_{n}$ is denoted $\ell(i, j, k)$. The point at which the ray enters $V_{n}$ is denoted $\mathbf{p}_{i, j, k}$. We also let $\mathbf{p}_{i, j, K_{i, j}+1}$ be the point at which the ray exits the domain.

We let

$$
\psi_{g}[i, j, k]=\psi_{g}\left(\mathbf{p}_{i, j, k}, \hat{\Omega}_{i}\right),
$$

though we note that the algorithms only need to store these quantities for $k=1$. For this reason we set

$$
\psi_{g}[i, j]=\psi_{g}[i, j, 1]
$$

This is the angular flux in direction $\hat{\Omega}_{i}$ at the point at which the $j^{\text {th }}$ trajectory for $\hat{\Omega}_{i}$ first intersects the domain.

2.2. Integral Approximations. If the trajectories have been chosen appropriately then for any $n$ and any $i$, we can consider all trajectories in direction $\hat{\Omega}_{i}$ that intersect $V_{n}$, and we can form the slice of each trajectory cyclinder whose axis is the intersection of the trajectory with $V_{n}$. These cylinder slices should roughly partition $V_{n}$. This allows us to approximate the integral of a function $f$ defined on $V_{n}$ as follows:

$$
\int_{V_{n}} f(\vec{r}, \hat{\Omega}) d V \approx \sum_{j=1}^{J_{i}} \sum_{\substack{k \\ 1 \leq k \leq K \\ \iota(i, j, k)=n}} \Delta S_{i, j} \int_{0}^{\ell(i, j, k)} f\left(\mathbf{p}_{i, j, k}+t \hat{\Omega}\right) d t
$$

Note that, for any $i$ and $j$, the sum over $k$ consists of at most 1 term; if it consists of 0 terms it is considered to be 0 . 
If we apply this to the function $f \equiv 1$, the left hand side of $(9)$ is precisely $\operatorname{vol}[n]$, and we define the right hand side to be $\operatorname{vol}^{\prime}[i, n]$, i.e.,

$$
\operatorname{vol}[n] \approx \sum_{j=1}^{J_{i}} \sum_{\substack{k \\ 1 \leq k \leq K_{i, j} \\ \iota(i, j, k)=n}} \ell(i, j, k) \Delta S_{i, j} \equiv \operatorname{vol}^{\prime}[i, n] .
$$

In fact, we can use (10) to improve the accuracy of the approximation made in (9), as follows:

$$
\int_{V_{n}} f(\vec{r}, \hat{\Omega}) d V \approx \frac{\operatorname{vol}[n]}{\operatorname{vol}^{\prime}[i, n]} \sum_{j=1}^{J_{i}} \sum_{\substack{k \\ 1 \leq k \leq K_{i, j} \\ \iota(i, j, k)=n}} \Delta S_{i, j} \int_{0}^{\ell(i, j, k)} f\left(\mathbf{p}_{i, j, k}+t \hat{\Omega}\right) d t
$$

In particular, approximation (11) is precise for $f \equiv 1$.

2.3. Boundary Condition. The behavior at the boundary of the modeled domain is specified by real numbers $\rho\left(i, j ; i^{\prime}, j^{\prime}\right)\left(1 \leq i, i^{\prime} \leq I, 1 \leq j \leq J_{i}, 1 \leq j^{\prime} \leq J_{i^{\prime}}\right)$. These numbers specify how neutrons exiting the domain along trajectory $j$ of angular region $i$ re-enter the domain. A neutron traveling along a path within $\Delta \hat{\Omega}_{i} \Delta S_{i, j}$ may re-enter along a path within $\Delta \hat{\Omega}_{i^{\prime}} \Delta S_{i^{\prime}, j^{\prime}}$ with probability

$$
\rho\left(i, j ; i^{\prime}, j^{\prime}\right) \text {. }
$$

If the boundary is purely reflective (no neutrons are lost), then for any $i, j$,

$$
\sum_{i^{\prime}, j^{\prime}} \rho\left(i, j ; i^{\prime}, j^{\prime}\right)=1
$$

If the boundary is a vaccum (neutrons never return), then $\rho \equiv 0$.

\section{Outer Algorithm}

We now turn to the detailed description of the MOC algorithm for solving equation (2). The parameters that are considered inputs to the algorithm and are not modified at any time by the algorithm are listed in Figure 2.

The high-level pseudcode for the MOC algorithm is given in Figure 3. This is called the outer algorithm because its convergence loop is the outer-most loop in the MOC algorithm. The outer algorithm relies on subroutines, described in subsequent sections, which rely on their own convergence loops.

The outer algorithm is provided with all of the global constants, together with initial guesses for $K_{\text {eff }}$, the values of $\psi$ on the boundary of the domain, and the values of $\phi$ throughout the domain. These guesses determine values for the fission component of the source, i.e., for

$$
Q_{g}^{f}(\vec{r})=\frac{\chi_{g}}{K_{\text {eff }}} \sum_{g^{\prime}} \nu \sigma_{g^{\prime}}^{f}(\vec{r}) \phi_{g^{\prime}}(\vec{r}) .
$$

The $Q_{g}^{f}$ are integrated over space and energy to yield a single scalar quantity called the total fission. Now, equation (8) can be written

$$
\left[\hat{\Omega} \cdot \vec{\nabla}+\sigma_{g}(\vec{r})\right] \psi_{g}(\vec{r}, \hat{\Omega})=\sum_{g^{\prime}} \sigma_{g g^{\prime}}^{s}(\vec{r}) \phi_{g^{\prime}}(\vec{r})+Q_{g}^{f}(\vec{r}) .
$$


symbol meaning

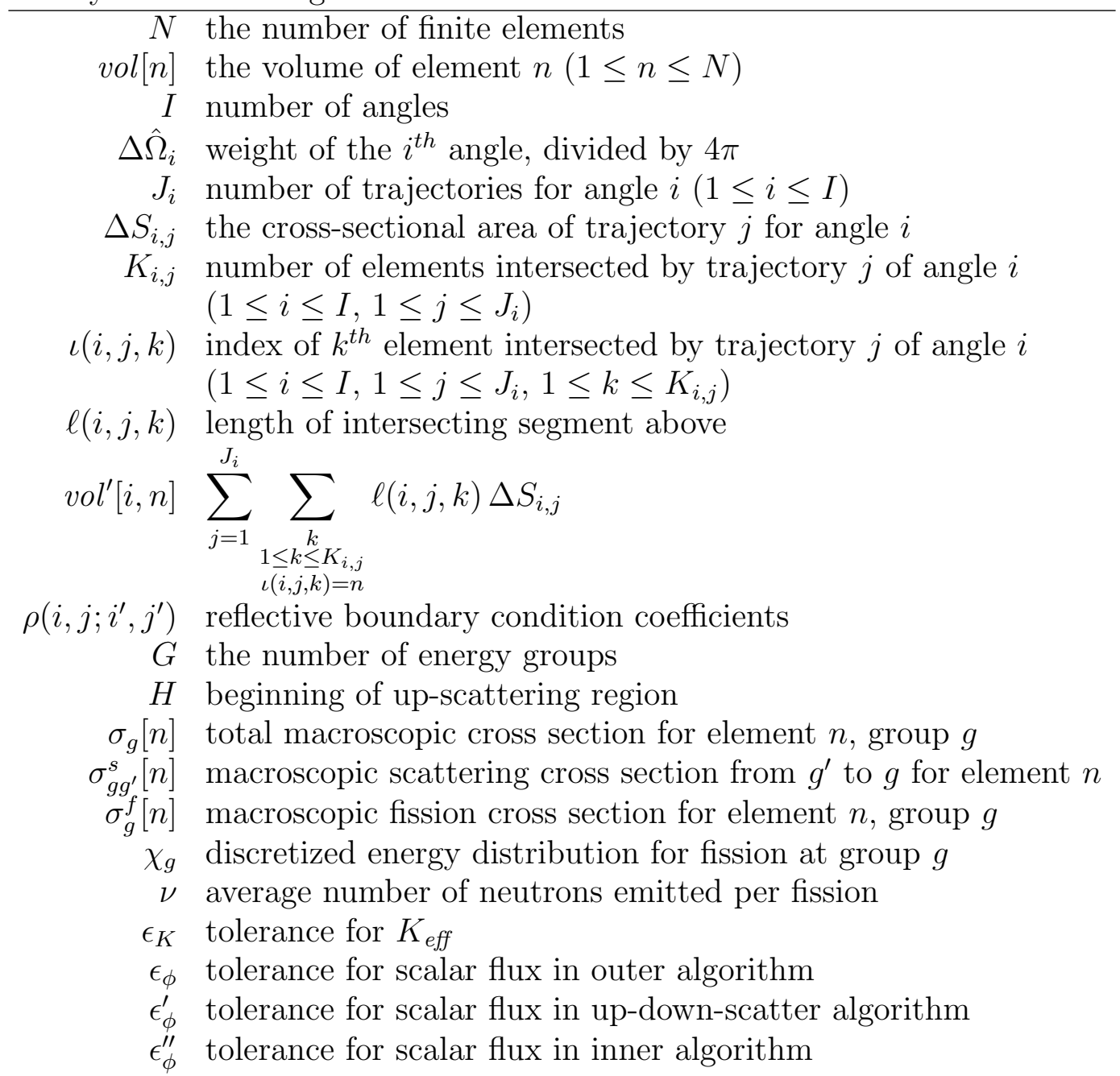

Figure 2. Global parameters used by all algorithms

Considering the $Q_{g}^{f}$ to be fixed, we arrive at a system of $G$ equations that must be solved simultaneously for $\psi$ (on the boundary) and $\phi$ (throughout). We will discuss shortly the procedure for solving this system. Once an approximate solution has been achieved, the new values for $\phi$ are used to calculate new values for the $Q_{g}^{f}$, and the total fission is re-computed. The ratio between the new and old values for the total fission is used to update the estimate for $K_{\text {eff }}$, and we proceed to solve the new simultaneous system. Iteration stops when the rates of change of $K_{\text {eff }}$ and of $\phi$ fall below specified tolerances.

\section{Middle Algorithms}

We now turn to the "middle" algorithms that are used to solve the simultaneous systems described above. Typically, the scattering matrix $\left(\sigma_{g g^{\prime}}^{s}\right)$ is "largely" lower triangular. That is, there is some $H(1 \leq H \leq G)$, usually close to $G$, such that $\sigma_{g g^{\prime}} \equiv 0$ whenever $g<H$ and $g^{\prime}>g$. (This reflects the fact that, for the most part, the neutrons tend to lose energy after scattering, except perhaps in the very low end of the energy spectrum.) In this "downscattering only" region, the system of equations can be solved sequentially: equation (12) 

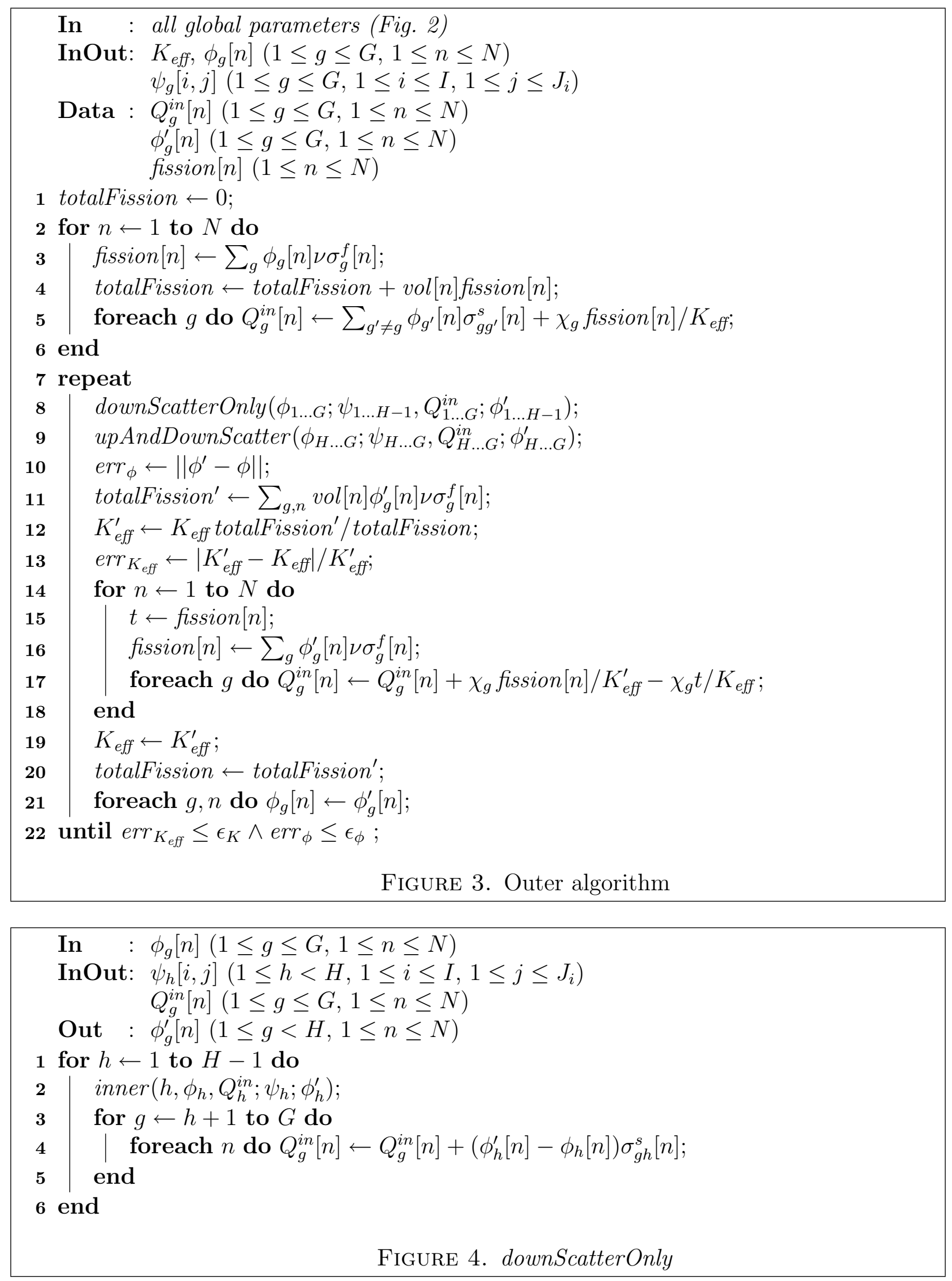


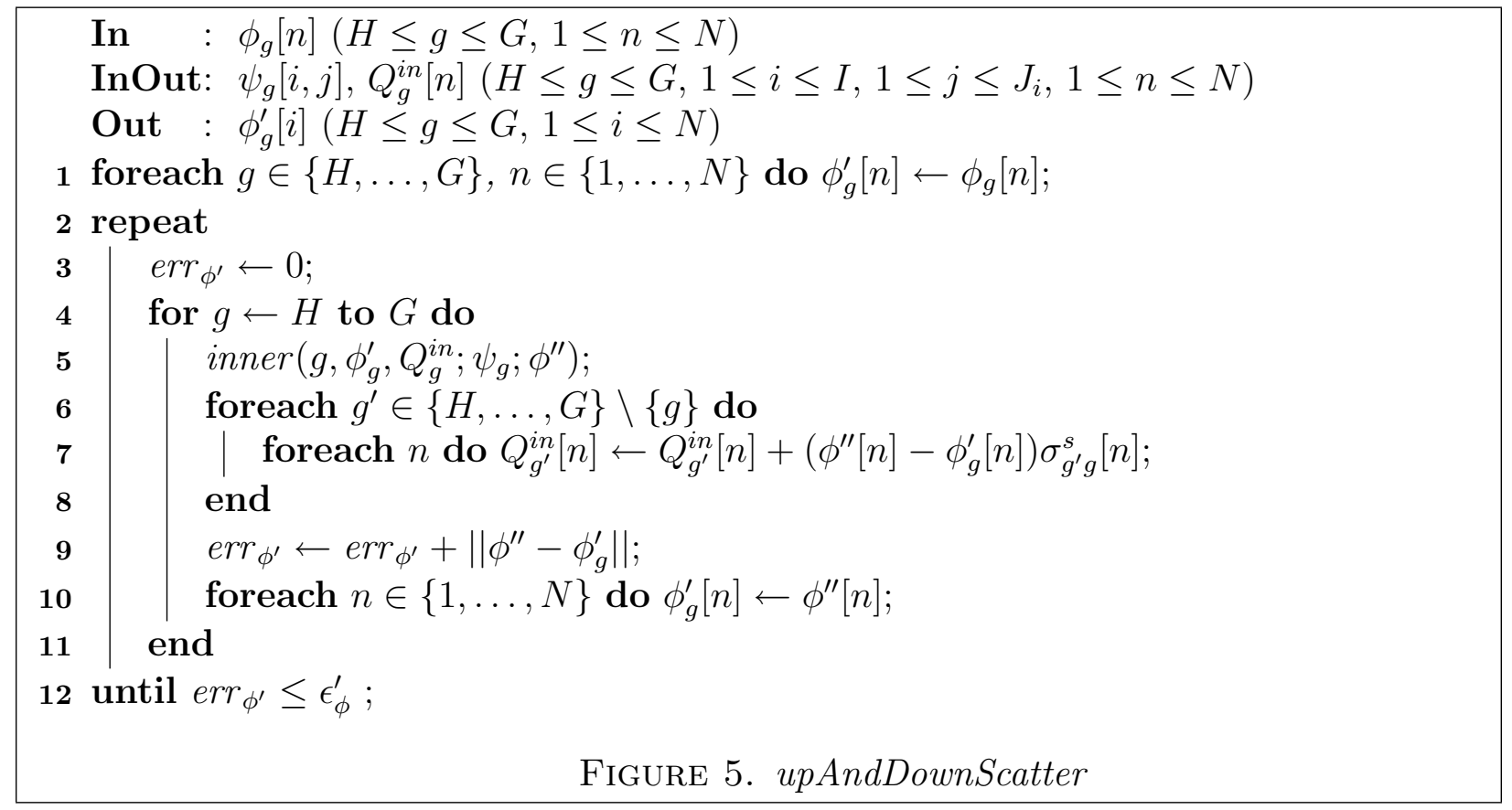

for $g=1$ involves only $\psi_{1}$ and $\phi_{1}$, and not any $\psi_{k}$ or $\phi_{k}$ for $k>1$. (We will discuss shortly the subroutine for solving this equation.) The solutions for $g=1$ can then be substituted into equation (12) for $g=2$, which then involves only $\psi_{2}$ and $\phi_{2}$, which is then solved, and so on. The precise algorithm dealing with the down-scattering-only region is given in Figure 4.

For the region $g \geq H$, where up and down scattering are possible, an iteration scheme is used. This algorithm is described in Figure 5.

\section{InNER Algorithm}

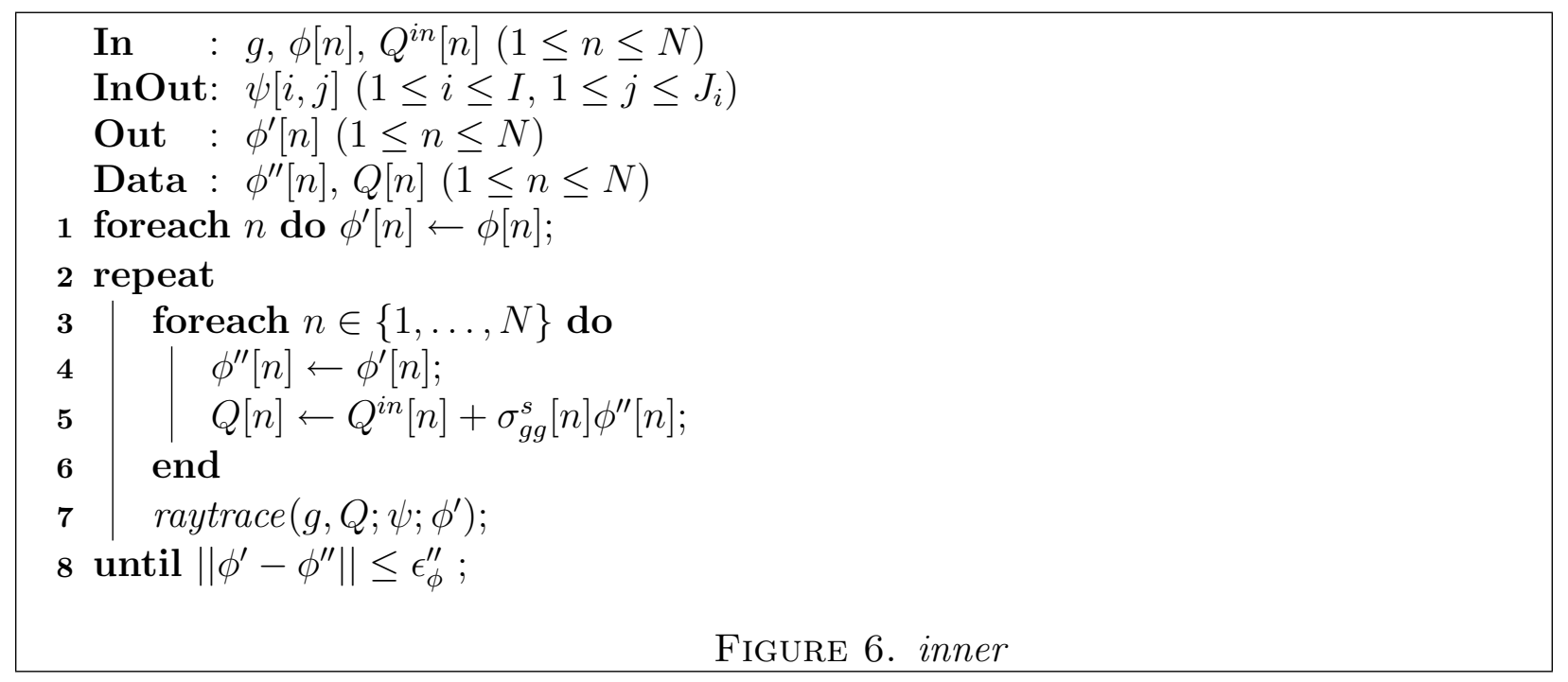


Both of the middle algorithms rely on a subroutine to solve an equation involving only one group $g$. Let

$$
Q_{g}^{i n}(\vec{r})=\sum_{g^{\prime} \neq g} \phi_{g^{\prime}}(\vec{r}) \sigma_{g g^{\prime}}^{s}(\vec{r})+\frac{\chi_{g}}{K_{e f f}} \sum_{g^{\prime}} \phi_{g^{\prime}}(\vec{r}) \nu \sigma_{g^{\prime}}^{f}(\vec{r}) .
$$

Then we can re-write (12) as the in-group equation

$$
\left[\hat{\Omega} \cdot \vec{\nabla}+\sigma_{g}(\vec{r})\right] \psi_{g}(\vec{r}, \hat{\Omega})=\phi_{g}(\vec{r}) \sigma_{g g}^{s}(\vec{r})+Q_{g}^{i n}(\vec{r}) .
$$

In (14), $Q_{g}^{i n}$ is considered fixed, and the problem is to solve for $\psi_{g}$ (on the boundary) and $\phi_{g}$ (throughout). The algorithm to do this is called the inner algorithm, and is described in Figure 6.

The inner algorithm begins with the current best estimate of $\phi_{g}$. Given this, we can compute the total source

$$
Q_{g}(\vec{r})=\phi_{g}(\vec{r}) \sigma_{g g}^{s}(\vec{r})+Q_{g}^{i n}(\vec{r}) .
$$

Equation (14) then becomes

$$
\left[\hat{\Omega} \cdot \vec{\nabla}+\sigma_{g}(\vec{r})\right] \psi_{g}(\vec{r}, \hat{\Omega})=Q_{g}(\vec{r}) .
$$

The problem now is to solve (16), treating the total source $Q_{g}$ as fixed. This is accomplished by the ray-tracing algorithm, which is described in detail below. The ray-tracing algorithm takes as input the current best estimate for $\psi_{g}$ on the boundary and the total source $Q_{g}$. It outputs the new boundary values of $\psi_{g}$, and the values of $\phi_{g}$ throughout the domain, approximately satisfying (16). The new values of $\phi_{g}(\vec{r})$ are used to update the total source $Q_{g}(\vec{r})$ using (15), and the ray-tracing technique is applied again. Iteration continues in this way until the relative change in $\phi_{g}$ between successive iterations falls below a specified tolerance.

\section{Ray-tracing Algorithm}

We now turn to the ray tracing algorithm for solving (16), the central technique of the MOC. We will drop the subscript $g$ to simplify the notation. The pseudocode for the algorithm is given in Figure 7.

The idea of the ray-tracing algorithm is to sample the domain with a set of rays that pass through the domain at various angles $\hat{\Omega}$ and that have various starting points. Restricted to a single ray and a single element, (16) becomes a simple first order ordinary differential equation in one variable, which can be solved analytically. One computes the solution along a ray by starting at the point at which the ray enters the boundary, where the value of $\psi$ is given, and propagating forward to compute the value of $\psi$ at successive elements intersected by the ray. As we shall see, the average scalar flux $\phi$ in an element can be approximated by a sum. Each ray passing through the element contributes one term to the sum. That term is a function of the value of $\psi$ where the ray enters the element and the value where the ray exits the element. After each new value of $\psi$ is computed, the contribution to the scalar flux is computed and added to the variable storing $\phi$ for that element. The incoming value of $\psi$ can then be forgotten. This is how the algorithm avoids storing $\psi$ at all elements.

The analytical solution is obtained as follows. Assume that

$$
\mathbf{p}=\left(x_{0}, y_{0}, z_{0}\right) \in \mathbb{R}^{3}
$$




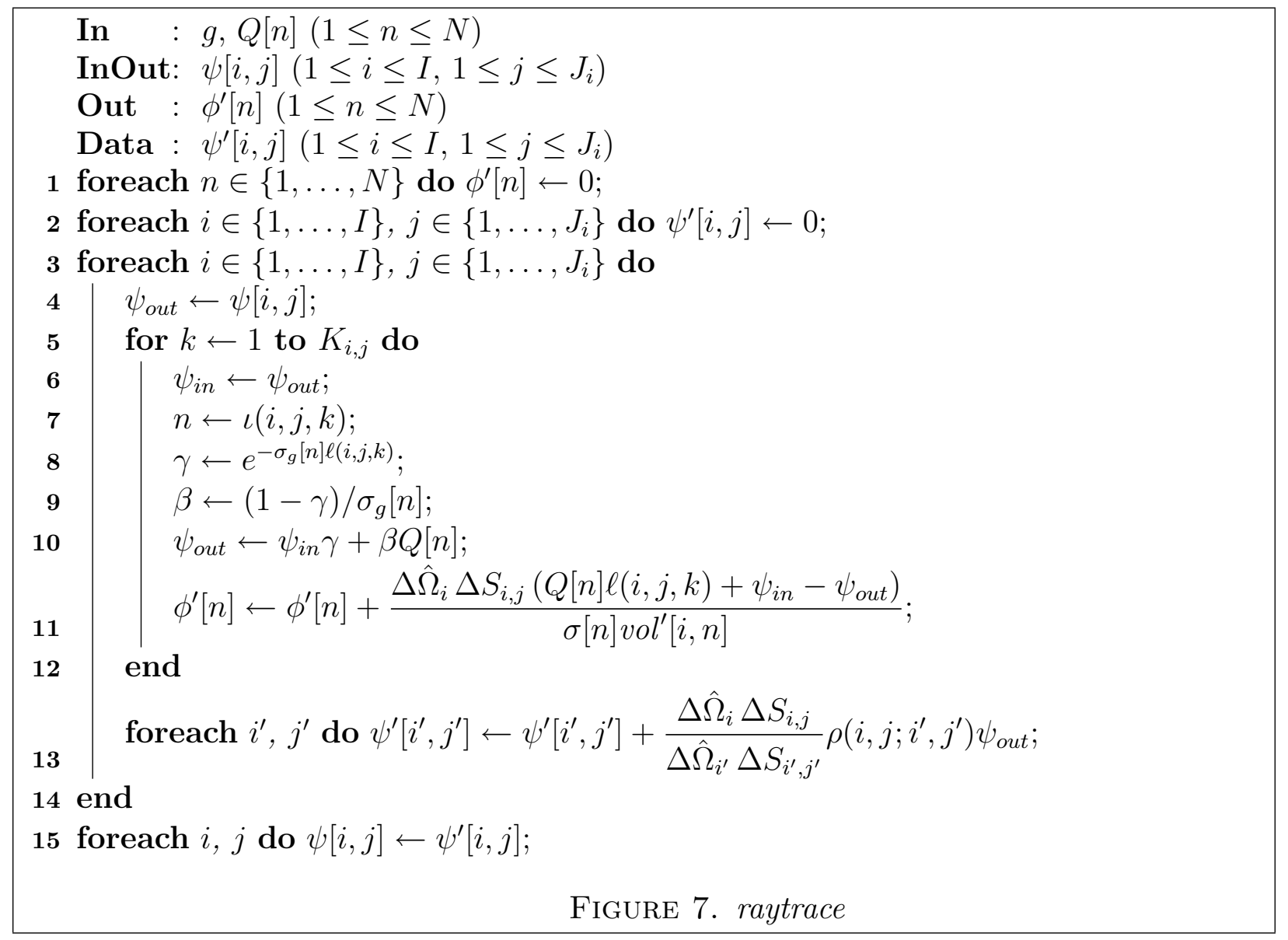

is the point at which a ray enters an element and that the ray is oriented in direction

$$
\hat{\Omega}=(u, v, w) \in S^{2} .
$$

Assume furthermore that we are given $\psi_{i n}$, the value of $\psi$ in direction $\hat{\Omega}$ at $\mathbf{p}$. We wish to compute $\psi_{\text {out }}$, the value of $\psi$ in direction $\hat{\Omega}$ at the point at which the ray exits the element.

Let $\zeta: \mathbb{R} \rightarrow \mathbb{R}^{3} \times S^{2}$ be the function defined by

$$
\zeta(t)=(\mathbf{p}+\hat{\Omega} t, \hat{\Omega}) .
$$

Then

$$
\zeta(t)=((x(t), y(t), z(t)), \hat{\Omega})
$$

where

$$
x(t)=x_{0}+u t, \quad y(t)=y_{0}+v t, \quad z(t)=z_{0}+w t .
$$

Let $f=\psi \circ \zeta$. Note that $f(t)$ is the value of $\psi$ in direction $\hat{\Omega}$ at the point a distance of $t$ along the ray from $\mathbf{p}$. If the length of the segment of the ray intersecting the element is $l$, then $f(l)=\psi_{\text {out }}$.

From the chain rule, we have

$$
f^{\prime}(t)=\frac{\partial \psi}{\partial x} \frac{\partial x}{\partial t}+\frac{\partial \psi}{\partial y} \frac{\partial y}{\partial t}+\frac{\partial \psi}{\partial z} \frac{\partial z}{\partial t}=u \frac{\partial \psi}{\partial x}+v \frac{\partial \psi}{\partial y}+w \frac{\partial \psi}{\partial z}=[\hat{\Omega} \cdot \vec{\nabla} \psi](\zeta(t)) .
$$


Within a single element, we assume that the source $Q$ and cross section $\sigma$ are constant, so (16) becomes

$$
f^{\prime}(t)+\sigma f(t)=Q
$$

The unique solution to (18) satisfying $f(0)=\psi_{\text {in }}$ is

$$
f(t)=\psi_{i n} e^{-\sigma t}+\frac{Q\left(1-e^{-\sigma t}\right)}{\sigma} .
$$

Substituting $l$ for $t$ in (19) gives the desired closed-form expression for $\psi_{\text {out }}$ :

$$
\psi_{\text {out }}=\psi_{\text {in }} e^{-\sigma l}+\frac{Q\left(1-e^{-\sigma l}\right)}{\sigma} .
$$

We now turn to the question of the computation of $\phi$ at all points in the domain. Integrating both sides of (18) from 0 to $l$, and applying the Fundamental Theorem of Calculus, we obtain

$$
\psi_{\text {out }}-\psi_{\text {in }}+\sigma \int_{0}^{l} f(t) d t=Q l
$$

Hence

$$
\int_{0}^{l} f(t) d t=\frac{Q l-\psi_{\text {out }}+\psi_{\text {in }}}{\sigma} .
$$

By definition,

$$
\begin{aligned}
\phi[n] & =\frac{1}{\operatorname{vol}[n]} \int_{V_{n}} \phi(\vec{r}) d V \\
& =\frac{1}{4 \pi \operatorname{vol}[n]} \int_{V_{n}} \int_{S^{2}} \psi(\vec{r}, \hat{\Omega}) d \hat{\Omega} d V \\
& =\frac{1}{4 \pi v o l[n]} \int_{S^{2}} \int_{V_{n}} \psi(\vec{r}, \hat{\Omega}) d V d \hat{\Omega} \\
& \approx \frac{1}{\operatorname{vol}[n]} \sum_{i=1}^{I} \Delta \hat{\Omega}_{i} \int_{V_{n}} \psi\left(\vec{r}, \hat{\Omega}_{i}\right) d V .
\end{aligned}
$$

Now for any $i$, we can apply (11) to the function $\psi\left(-, \hat{\Omega}_{i}\right)$ restricted to $V_{n}$ and then use (22) to obtain

$$
\begin{aligned}
\int_{V_{n}} \psi\left(\vec{r}, \hat{\Omega}_{i}\right) d V & \approx \frac{v o l[n]}{\operatorname{vol}^{\prime}[i, n]} \sum_{j=1}^{J_{i}} \sum_{\substack{k \leq k \\
1 \leq k \leq K_{i, j} \\
\iota(i, j, k)=n}} \Delta S_{i, j} \int_{0}^{\ell(i, j, k)} \psi\left(\mathbf{p}_{i, j, k}+t \hat{\Omega}_{i}, \hat{\Omega}_{i}\right) d t \\
& =\frac{\operatorname{vol}[n]}{\operatorname{vol}^{\prime}[i, n]} \sum_{j=1}^{J_{i}} \sum_{\substack{k \\
1 \leq k \leq K_{i, j} \\
\iota(i, j, k)=n}} \Delta S_{i, j} \frac{Q \ell(i, j, k)-\psi[i, j, k+1]+\psi[i, j, k]}{\sigma}
\end{aligned}
$$


Substituting (24) into (23) yields

$$
\phi[n] \approx \sum_{i=1}^{I} \sum_{j=1}^{J_{i}} \sum_{\substack{1 \leq k \leq K_{i, j} \\ \iota(i, j, k)=n}} \frac{\Delta S_{i, j} \Delta \hat{\Omega}_{i}}{\sigma \operatorname{vol}^{\prime}[i, n]}(Q \ell(i, j, k)-\psi[i, j, k+1]+\psi[i, j, k])
$$

This is the formula that is used to update $\phi^{\prime}$ in line 11 of Figure 7.

\section{COnformance}

The algorithms keep track of the average scalar flux $\phi_{g}[n]$ for all elements $n$, and the angular flux $\psi$ on the boundary. To be precise, we let $\psi_{g}[i, j]$ denote the angular flux at the point at which trajectory $j$ of angular region $i$ enters the domain being modeled.

The main ("outer") algorithm is given in Figure 3. The input to this algorithm consists of all the quantities listed in Figure 2. This algorithm calls two subroutines, downScatterOnly and upAndDownScatter, described in Figures 4 and 5, respectively. Each of these subroutines calls inner, given in Figure 6, which solves equation (14) for a fixed energy group $g$.

An implementation is considered conformant with this specification if it performs the same computation as the algorithm of Figure 3. By "same computation," we mean the same if all arithmetic operations were carried out as (infinite precision) operations on real numbers. Hence computed results may differ due to the lack of associativity, commutativity, etc., of floating-point operations.

\section{REFERENCES}

[1] E. E. Lewis and Jr. W. F. Miller. Computational Methods of Neutron Transport. American Nuclear Society, Inc., La Grange Park, Illinois, USA, 1993. 


\section{Argonne}

\section{Mathematics and Computer Science Division}

Argonne National Laboratory

9700 South Cass Avenue, Bldg. 240

Argonne, IL 60439

www.anl.gov

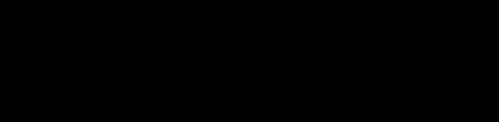

